\title{
Phase and intensity dependence of the dynamical Franz-Keldysh effect
}

\author{
C. J. Dent,${ }^{1}$ B. N. Murdin, ${ }^{2}$ and I. Galbraith ${ }^{1, *}$ \\ ${ }^{1}$ School of Engineering and Physical Sciences, David Brewster Building, Heriot-Watt University, Edinburgh EH14 4AS, United Kingdom \\ ${ }^{2}$ Department of Physics, University of Surrey, Guildford GU2 7XH, United Kingdom
}

(Received 25 November 2002; published 16 April 2003)

\begin{abstract}
We present theoretical results on the nonlinear optics of semiconductor quantum wells in intense $\mathrm{THz}$ electric fields (the dynamic Franz-Keldysh effect or DFKE). The absorption spectra show a rich variety of behavior, including $\mathrm{THz}$ replicas of the $2 p$ exciton and $\mathrm{THz}$ sidebands of the $1 s$ exciton. We calculate the dependence of these features on the phase and intensity of the $\mathrm{THz}$ field using the extended semiconductor Bloch equations, and discuss the relevance of our results to future experiments. The $1 s$-sideband absorption feature shows a strong dependence on the phase of the THz field, and phase averages to zero. We also discuss the relative advantages and disadvantages of reflectivity and absorption spectroscopies for probing the DFKE.

DOI: 10.1103/PhysRevB.67.165312

PACS number(s): 78.40.Fy, 71.35.Cc, 78.67.De
\end{abstract}

\section{INTRODUCTION}

The Franz-Keldysh effect (FKE), i.e., the modification of optical absorption spectra by an applied electric field, has been known for many years. ${ }^{1,2}$ Subsequently the generalization of the FKE to time-dependent electric fields, the dynamical Franz-Keldysh effect (DFKE) has also been studied. ${ }^{3}$ The quantity which characterizes the crossover between the FKE and DFKE regimes is the ratio $\gamma$ between the ponderomotive energy (i.e., the average kinetic energy of a particle of mass $m$ and charge $e$ in an electric field $F_{0} \cos \omega t$ ),

$$
E_{\text {pon }}=\frac{e^{2} F_{0}^{2}}{4 m \omega^{2}}
$$

and the photon energy $\hbar \omega$. The problem may be treated in terms of multiphoton processes for $\gamma \ll 1$ or in terms of the FKE for $\gamma \gg 1$. The DFKE corresponds to $\gamma \sim 1$. In addition, some manifestations of the DFKE are most noticeable when the frequency of the driving field is near resonance with a significant dipole-allowed transition.

In atomic physics, transition energies are typically 1 $-10 \mathrm{eV}$. For photon energies of this order, very high field strengths are necessary to obtain $\gamma \sim 1$. Recent theoretical and experimental effort has been devoted to the DFKE in semiconductor systems, where the internal transitions of excitons (i.e., hydrogenlike bound states of electron-hole pairs) are in the THz (terahertz) regime (a frequency of $1 \mathrm{THz}$ is equivalent to a photon energy of approximately $4.1 \mathrm{meV}$ ). For a photon energy near resonance with the $1 s-2 p$ exciton transition, $\gamma \sim 1$ may be obtained with $F \sim 1 \mathrm{kV} / \mathrm{cm}$, an intensity which is now routinely available from sources such as free-electron lasers.

In the initial work in semiconductor quantum wells, ${ }^{4}$ the Coulomb interaction was neglected, and it was calculated that the DFKE blue shifts the main band-edge absorption step by the ponderomotive energy, and increases the belowgap absorption. Subsequent studies have included the Coulomb interaction, and a rich variety of behavior has been predicted. It was shown both theoretically and experimentally in InGaAs quantum wells that the DFKE manifests itself as a blue shift in the exciton peak. ${ }^{5}$ Other results include the distribution of the resulting anisotropic wave packet and the $\mathrm{THz}$ emission spectrum, ${ }^{6}$ and the dependence of the DFKE on the polarization of the THz field. ${ }^{7}$ Theoretical work on the DFKE has been based on either Green's function techniques, ${ }^{4,5,7}$ or the conceptually simpler extension of the well-known semiconductor Bloch equations (SBE's) (Ref. 8) to include the THz field. ${ }^{6,9}$

Typically the DFKE is measured using pump-probe transmission experiments. In this paper we model such a setup having a $\mathrm{cW}-\mathrm{THz}$ pump beam and an ultrashort interband probe pulse. The absorption of the probe beam in the presence of the pump is then explored as a function of various pump beam parameters. In Sec. II we outline the derivation of the extended SBE's and compare results obtained from them with earlier Green's function calculations. Intuitively, one expects the phase of the $\mathrm{THz}$ field to be significant as its oscillation period ( $\sim 500 \mathrm{fs})$ is much longer than the 50 -fs probe pulse; in Sec. III we show the dependence of the various features in the absorption spectrum on the phase of the $\mathrm{THz}$ field at the probe time. In Sec. IV we present further new results on the intensity dependence of the below-gap absorption features, and then in Sec. V we discuss the appearance of the DFKE in reflectivity spectra. Finally, in Sec. VI we present our conclusions, including the prospects for confirming our predictions in experiment.

\section{THEORETICAL MODEL}

As the interband probe field, $\varepsilon(\omega)$, is weak, its absorption spectrum may be calculated from the polarization it induces in the sample as

$$
\alpha(\omega) \propto \operatorname{Im}\left(\frac{P(\omega)}{\varepsilon(\omega)}\right),
$$

where $\varepsilon(t)$ is the probe field. The polarization is given in the Hartree-Fock approximation by the semiconductor Bloch equations. The SBE's without the THz field terms are derived from the Hamiltonian ${ }^{8}$ 


$$
\begin{aligned}
H= & \sum_{\mathbf{k}}\left[E_{e, k} \alpha_{\mathbf{k}}^{\dagger} \alpha_{\mathbf{k}}+E_{h, k} \beta_{-\mathbf{k}}^{\dagger} \beta_{-\mathbf{k}}\right] \\
& -\sum_{\mathbf{k}}\left[\varepsilon(t) d_{c v} \alpha_{\mathbf{k}}^{\dagger} \beta_{-\mathbf{k}}^{\dagger}+\text { H.c. }\right] \\
& +\frac{1}{2} \sum_{\mathbf{k}, \mathbf{k}^{\prime}, \mathbf{q} \neq \mathbf{0}} V_{q}\left[\alpha_{\mathbf{k}+\mathbf{q}}^{\dagger} \alpha_{\mathbf{k}^{\prime}-\mathbf{q}}^{\dagger} \alpha_{\mathbf{k}^{\prime}} \alpha_{\mathbf{k}}\right. \\
& \left.+\beta_{\mathbf{k}+\mathbf{q}}^{\dagger} \beta_{\mathbf{k}^{\prime}-\mathbf{q}}^{\dagger} \beta_{\mathbf{k}^{\prime}} \beta_{\mathbf{k}}-2 \alpha_{\mathbf{k}+\mathbf{q}}^{\dagger} \beta_{\mathbf{k}^{\prime}-\mathbf{q}}^{\dagger} \beta_{\mathbf{k}^{\prime}} \alpha_{\mathbf{k}}\right]
\end{aligned}
$$

where $\alpha_{\mathbf{k}}$ and $\beta \mathbf{k}$ are the electron and hole operators, respectively, $d_{c v}$ is the interband dipole matrix element, $E_{(e, h), k}$ is the electron and hole band dispersions, and $V_{q}$ 's the Coulomb potential. The interband polarization is expressed as the sum $P=d_{c v} \Sigma_{\mathbf{k}} P_{\mathbf{k}}$, where $P_{\mathbf{k}}=\left\langle\beta_{-\mathbf{k}} \alpha_{\mathbf{k}}\right\rangle$.

The equations of motion for the $P_{\mathbf{k}}$ 's and the electron and hole occupation numbers may be obtained from the Heisenberg equations of motion for the electron and hole operators. The Coulomb terms in the Hamiltonian couple two-operator correlation functions to four-operator functions. A closed set of equations (the SBE's) may be obtained from the random phase approximation factorization of these four operator functions. ${ }^{8}$ The $\mathrm{THz}$ field is included via an additional intraband dipole term in the Hamiltonian as described in the Appendix. The resulting extended SBE's are then ${ }^{9}$

$$
\begin{gathered}
\frac{\partial P_{\mathbf{k}}}{\partial t}=-i\left(e_{e \mathbf{k}}+e_{h \mathbf{k}}\right) P_{\mathbf{k}}-\frac{e}{\hbar} \mathbf{F}_{\mathrm{THz}} \cdot \nabla_{\mathbf{k}} P_{\mathbf{k}} \\
-i\left(n_{e \mathbf{k}}+n_{h \mathbf{k}}-1\right) \Omega_{\mathbf{k}}+\left.\frac{\partial P_{\mathbf{k}}}{\partial t}\right|_{\text {coll }}, \\
\frac{\partial n_{(e, h) \mathbf{k}}}{\partial t}=-\frac{e}{\hbar} \mathbf{F}_{\mathrm{THz}} \cdot \nabla_{\mathbf{k}} n_{(e, h) \mathbf{k}}-2 \operatorname{Im}\left(\Omega_{\mathbf{k}} P_{\mathbf{k}}^{*}\right)+\left.\frac{\partial n_{(e, h) \mathbf{k}}}{\partial t}\right|_{\text {coll }} .
\end{gathered}
$$

Here, $e_{\lambda \mathbf{k}}=\left(E_{\lambda \mathbf{k}}-\Sigma_{\mathbf{q}} V_{|\mathbf{k}-\mathbf{q}|} n_{\lambda \mathbf{q}}\right) / \hbar$ are the Coulomb renormalized electron and hole energies, $\Omega_{\mathbf{k}}=\left(d_{c v} \varepsilon(t)\right.$ $\left.+\sum_{\mathbf{q}} V_{|\mathbf{k}-\mathbf{q}|} P_{\mathbf{q}}\right) / \hbar$ is the generalized Rabi frequency, and $\partial P_{\mathbf{k}} /\left.\partial t\right|_{\text {coll }}$, etc. represent the terms due to higher-order Coulomb correlations between carriers. The $\mathrm{THz}$ field is $\mathbf{F}_{\mathrm{THz}}(t)=\mathbf{F}_{0} \cos \left(\omega_{\mathrm{THz}} t-\theta\right)$, where $\theta$ is the phase of the $\mathrm{THz}$ field. Although we refer throughout to the " $\mathbf{F}$ " field in these equations as the $\mathrm{THz}$ field, the equations themselves are equally valid for fields of any frequency significantly below resonance with the gap including static ones.

Without the $\mathrm{THz}$ field, in the 2D case the SBE's have cylindrical symmetry. The $\mathrm{THz}$ field breaks the symmetry, and it is thus necessary to solve the extended SBE's on a 2D grid of points in $\mathbf{k}$ space. It is convenient for numerical purposes to introduce a moving frame of reference $\mathbf{k}^{\prime}=\mathbf{k}$ $+(e / \hbar) \int{ }^{t} \mathbf{F}_{\mathrm{THz}}\left(t^{\prime}\right) d t^{\prime}$. The transformed equations are then formally the same as the zero-field SBEs, except that the band dispersions oscillate in $\mathbf{k}$ space. We also make the relaxation-time approximation for the polarization dephasing, $\partial P_{\mathbf{k}} /\left.\partial t\right|_{\text {coll }}=-P_{\mathbf{k}} / T_{\text {deph }}$. As we use a weak interband

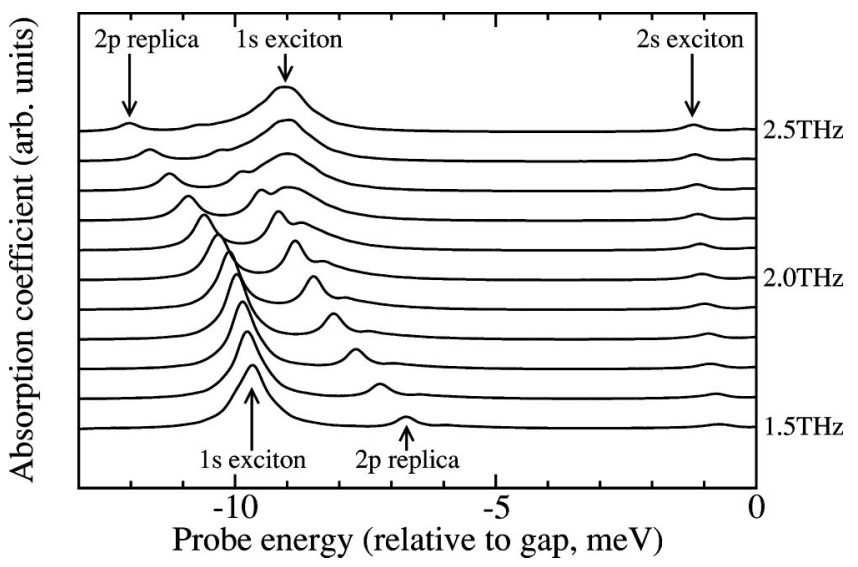

FIG. 1. Absorption spectra for $\mathrm{THz}$ frequencies (from bottom to top) $1.5,1.6, \ldots, 2.5 \mathrm{THz}$. The anti-crossing of the $1 \mathrm{~s}$ exciton peak, and the $2 p$ replica may be seen when the $\mathrm{THz}$ frequency equals the $1 s-2 p$ transition energy.

probe pulse in our calculations, the carrier populations are low, and we are justified in taking $\partial n_{(e, h) \mathbf{k}} /\left.\partial t\right|_{\text {coll }}=0$ and neglecting screening.

Throughout this paper, we use a two-band model of a semiconductor with GaAs parameters $\left(m_{e}=0.063 m_{0}, m_{h}\right.$ $=0.51 m_{0}, \varepsilon_{r}=13.0$, where $m_{0}$ is the free-electron mass.) We also introduce a form factor into the Coulomb potential to account for the finite width of the quantum well (taken as $12 \mathrm{~nm}$ ). These parameters give a $1 s$ exciton binding energy of $9.5 \mathrm{meV}(2.3 \mathrm{THz})$. We choose a dephasing time of $3 \mathrm{ps}$; this gives a $1 \mathrm{~s}$ linewidth of $\sim 0.5 \mathrm{meV}$, equivalent to the very best available samples. Our interband probe is centered at time $t=0$, with FWHM $50 \mathrm{fs}$ and a central frequency resonant with the band edge.

One interesting effect of a $\mathrm{THz}$ field is the appearance in the absorption spectrum of signatures of otherwise dark exciton states. This was first demonstrated using Green's function methods. ${ }^{10}$ For instance a feature appears one $\mathrm{THz}$ photon energy below the $2 p$ state due to the possibility of creating a $2 p$ exciton by simultaneous absorption of one probe photon and one $\mathrm{THz}$ photon (the " $2 p$ replica" peak.) This feature and its anticrossing with the $1 s$ exciton peak are shown in Fig. 1. The energy difference between the $2 p$ replica and the $2 s$ peak is greater(less) than $\hbar \omega_{\mathrm{THz}}$ if $\omega_{\mathrm{THz}}$ is tuned above(below) the $1 s-2 p$ transition. This is due to the ac Stark shift of the $2 p$ state, which we shall discuss in more detail in Sec. IV. We also note that in the spectra with $\mathrm{THz}$ frequency $2.1 \mathrm{THz}$ there is a more complicated double peak at around $-9 \mathrm{meV}$. This appears to be due to a more complicated anticrossing phenomenon involving the replica of the continuum $p$ states.

\section{PHASE DEPENDENCE}

Near the band edge, there are two principle $\mathrm{THz}$ generated features. We have already mentioned the $2 p$ replica peak which is seen below the gap. The second is a sideband feature $^{10}$ which appears $2 \hbar \omega_{\mathrm{THz}}$ above the main $1 s$ peak. This feature corresponds to the absorption of a single inter- 


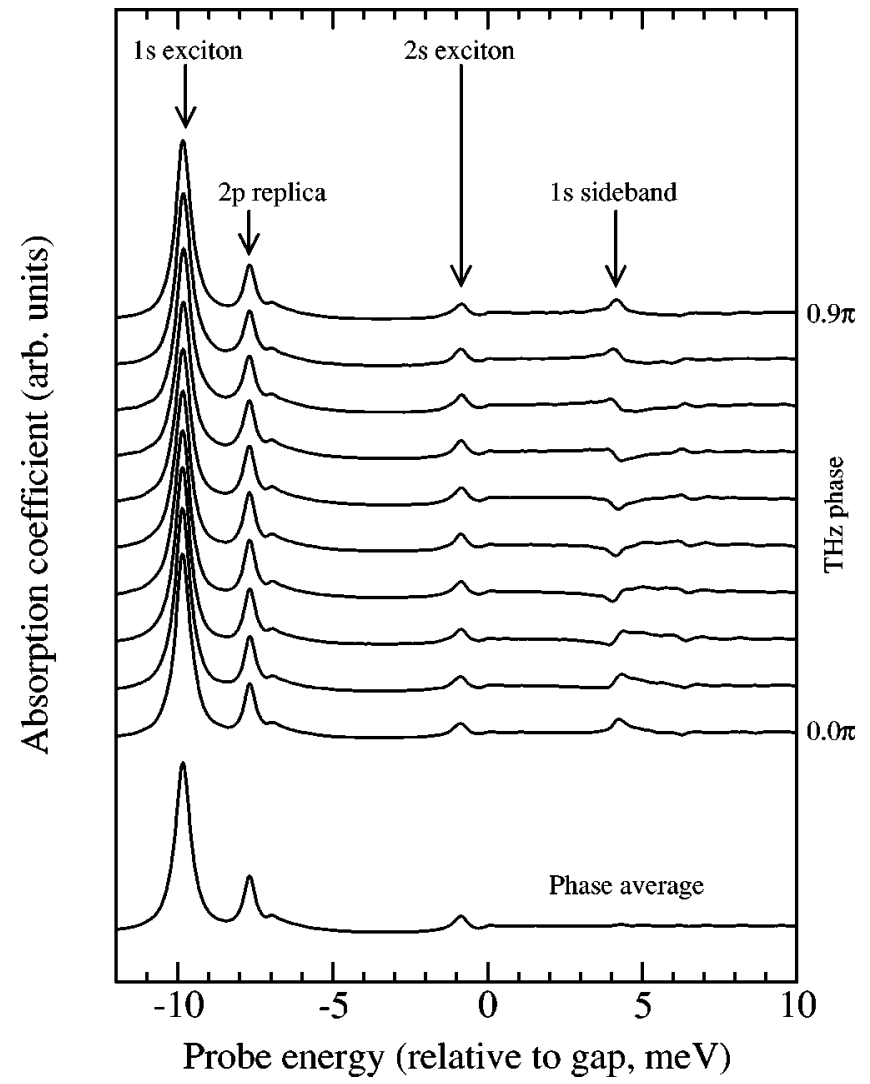

FIG. 2. Upper curves-absorption spectra for peak $\mathrm{THz}$ field 3 $\mathrm{kV} / \mathrm{cm}$ and $\mathrm{THz}$ frequency $1.7 \mathrm{THz}$. From bottom to top, the phase THz field is $0.0,0.1 \pi, \ldots, 0.9 \pi$. Lowest curves-average of spectra over phase for the same system parameters.

band photon together with the emission of two $\mathrm{THz}$ photons of opposite angular momentum.

The dependence of these features on the phase of the $\mathrm{THz}$ field at the time of the optical pulse is shown in Fig. 2. Varying the phase of the $\mathrm{THz}$ field causes a significant change in the shape of the sideband absorption feature, which evolves from a peak through to a dip. In contrast, the $2 p$ replica has very little phase dependence. The exciton peaks, while not changing shape or shifting, vary in height by about $15 \%$ as the phase changes, although this is hard to see in the figure. It is also worth noting that while the $1 \mathrm{~s}$ peak is strongest around phase $0.0 \pi$ and lowest around phase $0.5 \pi$, the reverse is true for the $2 s$ peak.

In DFKE experiments where the two fields are generated by different lasers, it may be difficult to lock the arrival of the probe pulse to a particular phase of the $\mathrm{THz}$ field. For a single measurement, one would therefore obtain a spectrum at an unknown phase. If, as usual, an average over a number of different individual experimental spectra is taken, then the results should be compared with phase-averaged theoretical predictions. We find (Fig. 2) that in our model the manifestations of the sideband in the continuum cancel on phase averaging, and the spectrum is then featureless in its vicinity. We thus expect the sideband feature to be very difficult to observe in transmission experiments (as opposed to luminescence ${ }^{11}$ ), unless it is possible to control or at least measure accurately the phase of the $\mathrm{THz}$ field.

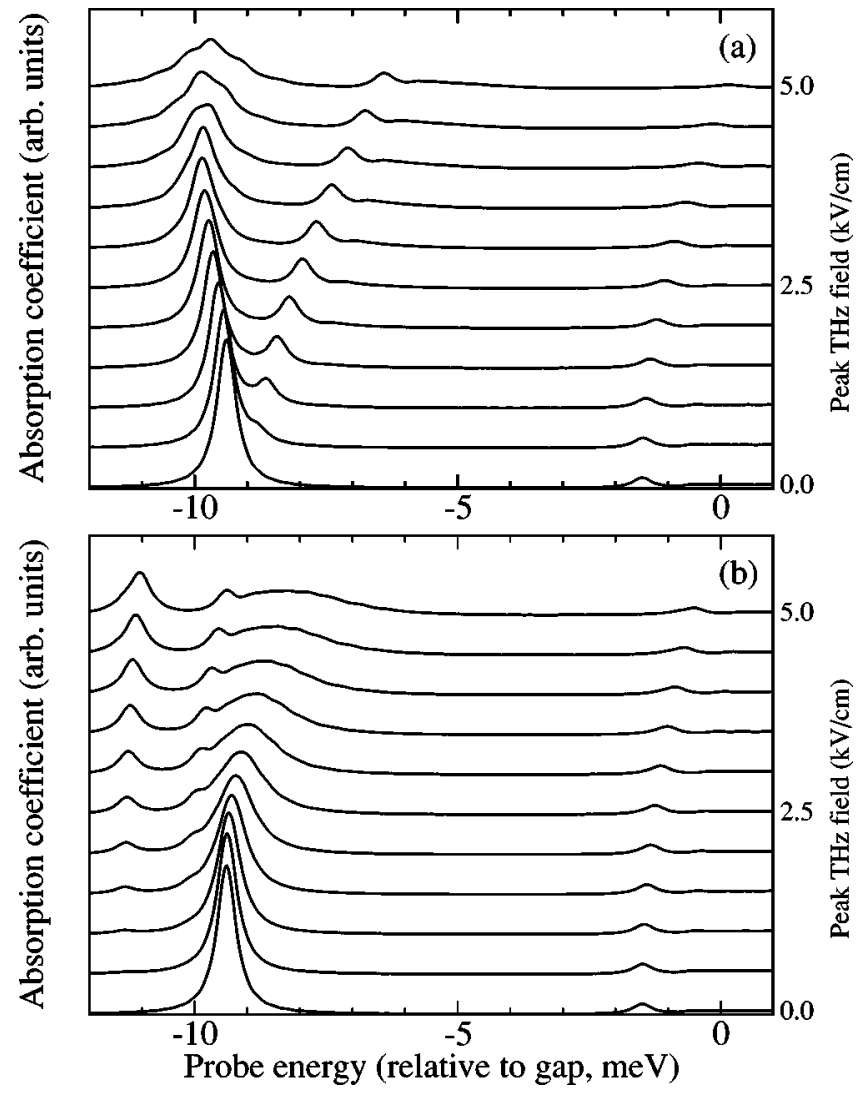

FIG. 3. (a) absorption spectra for THz frequency $1.7 \mathrm{THz}$. (b) absorption spectra for $\mathrm{THz}$ frequency $2.3 \mathrm{THz}$. In each case, the $\mathrm{THz}$ peak fields are, from bottom to top, $0.0,0.5,1.0, \ldots$, $5.0 \mathrm{kV} / \mathrm{cm}$.

\section{INTENSITY DEPENDENCE}

In Fig. 3 we show the dependence of the absorption spectrum on the THz intensity for two different $\mathrm{THz}$ frequencies. It has previously been demonstrated, in a system with greater broadening, ${ }^{5}$ that the main exciton peak shows qualitatively different behavior depending on whether the $\mathrm{THz}$ field is tuned above or below the $1 s-2 p$ transition. The effect of the DFKE itself is to blue shift all the main features. When $\omega_{\mathrm{THz}}$ is near resonant with the $1 s-2 p$ transition, the ac Stark shifts of those states are also significant. If the $\mathrm{THz}$ frequency is tuned above the $1 s-2 p$ transition, $\omega_{\mathrm{THz}}>\omega_{1 s-2 p}$, the ac Stark effect blue shifts the $1 s$ state and redshifts the $2 p$ state. If $\omega_{\mathrm{THz}}<\omega_{1 s-2 p}$, then the Stark shifts are in the opposite directions. Thus if $\omega_{\mathrm{THz}}>\omega_{1 s-2 p}$ the $1 s$ peak blue shifts for all $\mathrm{THz}$ intensities, while if $\omega_{\mathrm{THz}}<\omega_{1 s-2 p}$ the $1 s$ peak redshifts at low $\mathrm{THz}$ intensities, but as the intensity increases the DFKE dominates and a net blue shift occurs. ${ }^{5}$

With the short dephasing time we use, it is possible to investigate the intensity dependence of the other belowbandgap features. Both the $2 s$ and the dark $2 p$ exciton states are blue shifted by the DFKE at all THz intensities. As the $1 s-2 s$ transition is not dipole-allowed there is no significant Stark effect of the $2 s$ peak, but the $2 p$ state and its replica do undergo a Stark shift. If $\omega_{\mathrm{THz}}<\omega_{1 s-2 p}$, the Stark shift of the $2 p$ peak is in the blue direction, and the $2 p$ replica therefore blueshifts more than the $2 s$ peak, Fig. 3(a). Conversely, if 


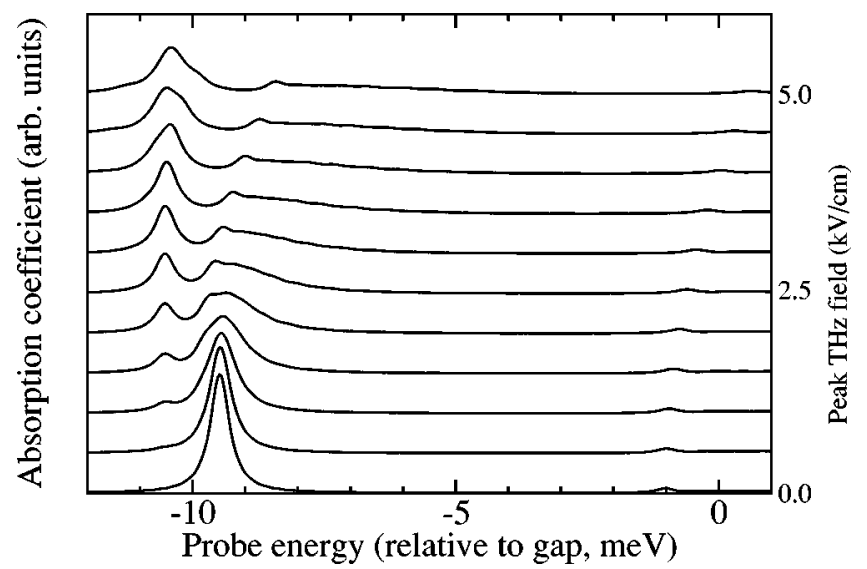

FIG. 4. Absorption spectra for $\mathrm{THz}$ frequency $2.3 \mathrm{THz}$ using a pure 2D model. The $\mathrm{THz}$ peak fields are $0.0,0.5,1.0, \ldots, \mathrm{kV} / \mathrm{cm}$ as in Fig. 3(b).

$\omega_{\mathrm{THz}}>\omega_{1 s-2 p}$, then the net blue shift of the $2 p$ replica is smaller than that of the $2 s$ peak, Fig. 3(b).

For the spectra plotted in Fig. 3, the $2 p$ replica and the $1 s$ exciton peaks may be resolved clearly. We observe that as the intensity increases, the $1 s$ peak broadens. This is presumably due to the THz-induced oscillations in the relative position of the bound electron and hole-when these are comparable in size with the exciton we expect the corresponding absorption feature to be significantly broadened. In analogy with Sec. I, we may quantify this by calculating the ratio $\alpha$ between the maximum displacement of a classical particle of mass $m$ in an applied field $F_{0} \cos \omega_{\mathrm{THz}} t$ with the corresponding 2D Bohr radius

$$
\alpha=\frac{e^{3} F_{0}}{2 \pi \hbar^{2} \omega^{2} \varepsilon_{0} \varepsilon} .
$$

For $\mathrm{THz}$ frequency $1.7 \mathrm{THz}$ and $\mathrm{GaAs}$ parameters we obtain $\alpha \simeq 0.4$ for $F_{0}=1.0 \mathrm{kV} / \mathrm{cm}, \alpha \simeq 1.3$ for $F_{0}=3.0 \mathrm{kV} / \mathrm{cm}$, and $\alpha \simeq 2.2$ for $F_{0}=5.0 \mathrm{kV} / \mathrm{cm}$. These values are consistent with our results, Fig. 3(a). The greater broadening at $2.3 \mathrm{THz}$, compared with $1.7 \mathrm{THz}$, may be accounted for as $2.3 \mathrm{THz}$ is approximately equivalent to the $1 s$ exciton binding energy, and therefore may actually break up the exciton.

Several authors have performed DFKE calculations using a purely two-dimensional Coulomb potential. One can obtain realistic exciton binding energies by adjusting the electron and hole effective masses. We show in Fig. 4 results obtained using the pure two-dimensional model (i.e. no form factor in the potential) and $\mathrm{THz}$ frequency $2.3 \mathrm{THz}$. We use the same dielectric constant as our other calculations, and we obtain the same $1 s$ binding energy by altering the effective masses to give a reduced mass of $0.0315 m_{e}$. The $n=2$ exciton states, and therefore the $2 p$ replica are at lower binding energies in the pure 2D model than the finite well-width model. We also find that the blue shifts in both the $1 s$ peak and the $2 p$ replica are smaller in these pure $2 \mathrm{D}$ calculations-the latter result is slightly surprising as $2 s$ peak actually shifts further in the pure 2D model than in the finite well-width model. This comparison shows that it is not

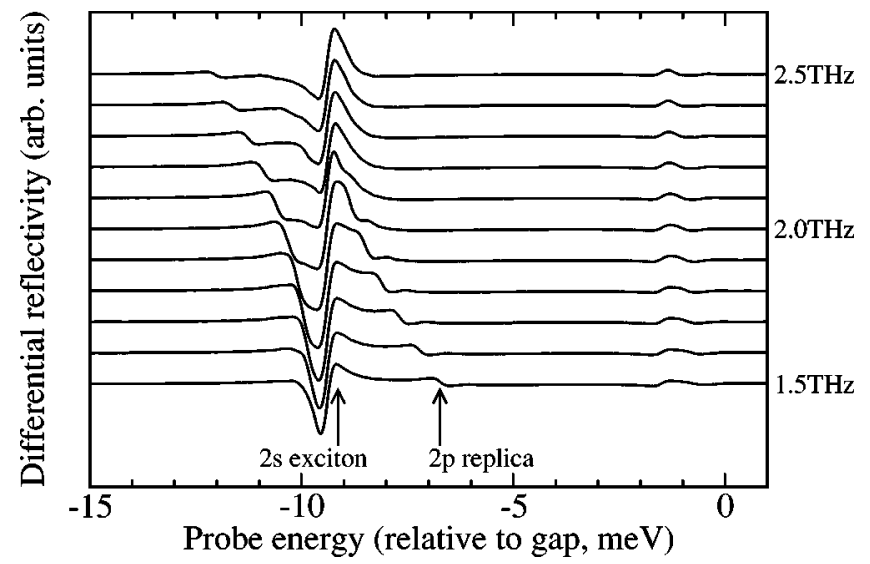

FIG. 5. Differential reflectivity spectra for different THz frequencies in a GaAs quantum well. The system parameters are the same as in Fig. 1. THz frequencies are, from bottom to top, $1.5,1.6, \ldots, 2.5 \mathrm{THz}$.

necessarily satisfactory to apply results obtained from pure 2D calculations when either quantitative predictions or direct comparison with experiment are required.

\section{REFLECTIVITY}

Thus far we have considered only the manifestation of the DFKE in absorption spectra as typically absorption measurements are straightforward to interpret. However, for GaAs/ AlGaAs quantum wells the substrate is absorbing and must be removed. To avoid any possible damage during this etching the possibility of using reflectivity measurements should be considered. In this section we compare some of our calculated absorption spectra with the corresponding reflectivity results. Reflectivity data are often more difficult to interpret for a variety of reasons. First, it usually has a lower signalnoise ratio and broader linewidths than transmission data due, among other factors, to multiple reflections in a multiwell sample, and to interface roughness. Second, and more fundamentally, where there is a simple peak in absorption, the corresponding feature in the reflectivity spectrum is a broader "hump dip." In practice, the clearest signal in reflectivity may often be obtained by measuring directly the differential reflectivity (DR), defined in our case as $R_{\mathrm{THz}}-R_{0}$, where $R_{\mathrm{THz}}$ and $R_{0}$ are the reflectivities with and without the $\mathrm{THz}$ field, respectively.

For small changes the deviation of the reflectivity from its background value may be calculated as

$$
R(\omega)-R_{\text {back }}(\omega) \propto \operatorname{Re}\left[\frac{P(\omega)}{\varepsilon(\omega)}\right] .
$$

Figure 5 shows differential reflectivity spectra calculated using the same system parameters as for the absorption spectra in Fig. 1. The various features are more difficult to resolve separately in DR than in absorption. Also, as features in DR are typically the difference between two hump dips they can take a variety of complicated shapes. Where it can be resolved separately, the $2 p$ replica appears as a shoulder on the main $1 s$ feature. Unlike in absorption, it is difficult to deter- 
mine accurately the energy of the features in the spectra, and to follow their evolution as the $\mathrm{THz}$ frequency changes.

We emphasize again that there are effects which broaden reflectivity spectra which are not as significant in absorption. In calculating all the spectra we have presented here, we have used precisely the same dephasing time, and have not allowed for any additional experimental broadening (in practice, additional broadening may be included by increasing the dephasing time, as doing so only increases linewidths without shifting any features). Therefore, the difference in ease of interpretation between absorption and DR is likely to be greater still in experiment than in the plots we have presented.

\section{CONCLUSIONS}

We have presented a detailed study of the effects of a $\mathrm{THz}$ electric field on the absorption spectrum of GaAs quantum wells, including the dependence of the various features on the phase of the THz field and on its intensity. The main below-gap features in the absorption spectra, namely, the $1 \mathrm{~s}$ peak and the single-photon THz replica of the $2 p$ peak, do not depend significantly on the phase of the THz field. However, the feature in the continuum corresponding to a sideband of the $1 s$ exciton, while not shifting, changes shape as the phase of the THz field varies. As a result, this feature will be difficult to observe in experiment unless the phase of the $\mathrm{THz}$ field can be closely controlled.

The DFKE itself blue shifts all the main absorption features at all THz intensities, but the direction of the ac Stark shifts depends on whether the $\mathrm{THz}$ field is tuned above or below the $1 s-2 p$ transition. The interplay between the two effects determines the direction and size of the shifts in the $1 s$ peak and the $2 p$ replica. We expect that it will prove easier to observe these features in transmission experiments rather than reflection, both due to the greater experimental broadening and the intrinsic spectral shapes in the latter. We also find that in order to make quantitative predictions for the sideband and replica features, it is necessary to include a finite well width in the calculations.

In order for the fine detail of the spectra, including the THz replica of the $2 p$ exciton, to be observed experimentally, a sample with very narrow $1 s$ exciton linear absorption linewidth (of the order of $1 \mathrm{meV}$ ) is required. In samples with greater broadening, the replica would manifest itself as a shoulder on, or an additional shift of, the $1 s$ peak. With the latest experimental technology and high quality samples, however, we are hopeful that it will prove possible to test our predictions.

\section{ACKNOWLEDGMENTS}

We acknowledge valuable discussions with C. R. Pidgeon, D. Clarke, and E. Abraham. This work was supported by grants from the UK EPSRC.

\section{APPENDIX: DERIVATION OF THE THZ TERM IN EQ. (5)}

In second quantization, the electric-field term in the Hamiltonian is

$$
H_{\text {Field }}=-e \mathbf{E} \cdot \int d \mathbf{r} \hat{\psi}^{\dagger}(\mathbf{r}) \mathbf{r} \hat{\psi}(\hat{\mathbf{r}})
$$

with $\mathbf{E}=\mathbf{E}_{\text {probe }}+\mathbf{F}_{\mathrm{THz}}$. Expanding the field operators in terms of Bloch states, $\hat{\psi}(\mathbf{r})=\Sigma_{\lambda=(c, v)} \psi_{\lambda \mathbf{k}}(\mathbf{r}) \hat{a}_{\lambda \mathbf{k}}$,

$$
H_{\text {Field }}=-e \mathbf{E} \cdot \sum_{\lambda \lambda^{\prime}=(c, v)} \sum_{\mathbf{k} \mathbf{k}^{\prime}} \mathbf{M}_{\mathbf{k k}^{\prime}}^{\lambda \lambda^{\prime}} \hat{a}_{\lambda \mathbf{k}}^{\dagger} \hat{a}_{\lambda^{\prime} \mathbf{k}^{\prime}},
$$

where $\mathbf{M}_{\mathbf{k} \mathbf{k}^{\prime}}^{\lambda \lambda^{\prime}}=\left\langle\lambda \mathbf{k}|\mathbf{r}| \lambda^{\prime} \mathbf{k}^{\prime}\right\rangle$ and $(c, v)$ denote the conduction and valence bands, respectively. The THz field makes a negligible contribution to the interband terms in the band index sum with $\lambda \neq \lambda^{\prime}$-these terms are the origin of the electricfield terms in the original SBE's. The THz field does, however make the dominant contribution to the intraband $\lambda$ $=\lambda^{\prime}$ terms. Replacing the conduction/valence band operators with electron/hole operators by the standard relations $\hat{a}_{c \mathbf{k}}=\alpha_{\mathbf{k}}$ and $\hat{a}_{v \mathbf{k}}=\beta_{-\mathbf{k}}^{\dagger}$, we obtain

$$
H_{\mathrm{THz}}=-e \mathbf{F}_{\mathrm{THz}} \cdot \sum_{\mathbf{k} \mathbf{k}^{\prime}}\left(\mathbf{M}_{\mathbf{k} \mathbf{k}^{\prime}}^{c c} \alpha_{\mathbf{k}}^{\dagger} \alpha_{\mathbf{k}^{\prime}}+\mathbf{M}_{\mathbf{k} \mathbf{k}^{\prime}}^{v v} \beta_{-\mathbf{k}} \beta_{-\mathbf{k}^{\prime}}^{\dagger}\right) .
$$

The THz part of the Heisenberg equation of motion for the electron number operator $\hat{n}_{e \mathbf{k}}=\alpha_{\mathbf{k}}^{\dagger} \alpha_{\mathbf{k}}$ is

$$
\begin{aligned}
\left.\hbar \frac{\partial \hat{n}_{e \mathbf{k}}}{\partial t}\right|_{\mathrm{THz}} & =-i\left[\hat{n}_{e \mathbf{k}}, H_{\mathrm{THz}}\right] \\
& =i e \mathbf{F}_{\mathrm{THz}} \cdot \sum_{\mathbf{k}^{\prime}}\left(\mathbf{M}_{k k^{\prime}}^{c c} \alpha_{\mathbf{k}}^{\dagger} \alpha_{\mathbf{k}^{\prime}}-\mathbf{M}_{k^{\prime} k}^{c c} \alpha_{\mathbf{k}^{\prime}}^{\dagger} \alpha_{\mathbf{k}}\right) .
\end{aligned}
$$

By taking expectation values the equation of motion for the occupation number $n_{e \mathbf{k}}=\left\langle\alpha_{\mathbf{k}}^{\dagger} \alpha_{\mathbf{k}}\right\rangle$ is obtained immediately.

It is now necessary to treat the matrix element $\mathbf{M}_{\mathbf{k k}}^{c c}$. As it stands, this is strictly ill defined as the position operator $\hat{\mathbf{r}}$ does not have the same periodicity as the lattice. ${ }^{12}$ However, making the approximation that the Bloch wave functions are plane waves and taking the continuum limit, one can write

$$
\mathbf{M}_{\mathbf{k} \mathbf{k}^{\prime}}^{c c}=-\frac{i}{L^{2}} \nabla_{\mathbf{k}^{\prime}} \int d \mathbf{r} e^{i\left(\mathbf{k}^{\prime}-\mathbf{k}\right) \cdot \mathbf{r}}=-i \nabla_{\mathbf{k}^{\prime}} \delta\left(\mathbf{k}-\mathbf{k}^{\prime}\right) .
$$

Expressions involving the gradient of the Dirac delta function under an integral may be evaluated, depending on the $\mathbf{k}$ and $\mathbf{k}^{\prime}$ dependence of the rest of the integrand, either by integration by parts, or by taking the gradient operator outside the integral. It must be emphasized that this representation of the matrix element only applies once the continuum limit has been taken, as the $\mathbf{k}$-space gradient operator is not defined on a discrete lattice of points in $\mathbf{k}$ space. The final result for the equation of motion of the occupation number is then

$$
\left.\hbar \frac{\partial n_{e \mathbf{k}}}{\partial t}\right|_{\mathrm{THz}}=-\frac{e \mathbf{F}_{\mathrm{THz}}}{\hbar} \cdot \nabla_{\mathbf{k}} n_{e \mathbf{k}} .
$$

Similar derivations apply for the hole occupation numbers and the $\mathbf{k}$ components of the polarization. 
*Electronic address: i.galbraith@hw.ac.uk

${ }^{1}$ W. Franz, Z. Naturforsch. A 13A, 484 (1958).

${ }^{2}$ L. Keldysh, Sov. Phys. JETP 34, 788 (1958) [Zh. Eksp. Teor. Fiz. 34, 1138 (1958)].

${ }^{3}$ Y. Yacoby, Phys. Rev. 169, 610 (1968).

${ }^{4}$ A.-P. Jauho and K. Johnsen, Phys. Rev. Lett. 76, 4576 (1996).

${ }^{5}$ K. Nordstrom, K. Johnsen, S. Allen, A.-P. Jauho, B. Birnir, J. Kono, T. Noda, H. Akiyama, and H. Sakaki, Phys. Rev. Lett. 81, 457 (1998).

${ }^{6}$ S. Hughes and D. Citrin, Phys. Rev. B 59, 5288 (1999).

${ }^{7}$ S. Hughes and D. Citrin, Phys. Rev. B 60, 13272 (1999).
${ }^{8} \mathrm{H}$. Haug and S. Koch, Quantum Theory of the Optical and Electronic Properties of Semiconductors, 3rd ed. (World Scientific, Singapore, 1994).

${ }^{9}$ T. Meier, G. von Plessen, P. Thomas, and S. Koch, Phys. Rev. Lett. 73, 902 (1994).

${ }^{10}$ K. Johnsen and A.-P. Jauho, Phys. Rev. Lett. 83, 1207 (1999).

${ }^{11}$ K. Nordstrom, K. Johnsen, S.J. Allen, Jr., A.-P. Jauho, B. Birnir, J. Kono, T. Noda, H. Akiyama, and H. Sakaki, Phys. Status Solidi B 204, 52 (1997).

${ }^{12}$ E. Blount, Solid State Phys. 13, 306 (1962). 